\title{
Psychotic traits in comedians
}

\author{
Victoria Ando, Gordon Claridge and Ken Clark
}

\section{Background}

The popular belief that creativity is associated with madness has increasingly become the focus of research for many psychologists and psychiatrists. However, despite being prime examples of creative thinking, comedy and humour have been largely neglected.
Aims
To test the hypothesis that comedians would resemble other creative individuals in showing a higher level of psychotic characteristics related to both schizophrenia and manic depression.

\section{Method}
A group of comedians $(n=500+)$ and a control sample of actors $(n=350+)$ completed an online questionnaire containing the short version of the Oxford-Liverpool

Inventory of Feelings and Experiences (O-LIFE), with scales measuring four dimensions of psychotic traits. Scores were compared with general population norms.

\section{Results}

Comedians scored significantly above O-LIFE norms on all four scales. Actors also differed from the norms but on only three of the scales. Most striking was the comedians' high score on both introverted anhedonia and extraverted impulsiveness.

\section{Conclusions}

This unusual personality structure may help to explain the facility for comedic performance.

\section{Declaration of interest}

\section{None.}

'There is always some frivolity in excellent minds; they have wings to rise, but also stray' (Joseph Joubert).

The age-old belief that creativity is associated with madness captures the public imagination and in recent years has increasingly become the focus of research for many professional psychologists and psychiatrists, who have provided substantial evidence to support the idea. ${ }^{2}$ A convincing interpretation of these findings suggests that the connection is mediated through an association with personality and cognitive traits that underlie and predispose to psychosis. ${ }^{3}$ This idea has been most commonly studied under the heading of 'schizotypy', denoting schizophrenia-like characteristics that can be observed widely in the general population, in the absence of symptoms of overt psychotic illness. It is now clear that the same formulation can be expanded to include the other major form of psychosis, bipolar disorder, with a corresponding broadening of terminology in the personality sphere to refer to psychotic traits in general. ${ }^{4}$ Almost all the work within the above theoretical framework has been concerned with conventional creative forms within the arts and sciences. In contrast, despite being prime examples of creative thinking, comedy and humour have been largely neglected. There has been the occasional clinical observation that the rate of psychiatric disturbance in comedians seems high; ${ }^{5}$ but, with rare exceptions ${ }^{6}$ - and then more concerned with humour appreciation - there has been little systematic research on the topic. Where personality in comedians has been studied, this has involved questionnaires such as the 'Big Five,' which does not cover psychotic traits. Outside this specifically 'madness/creativity' literature - in more general writings about humour - the issue of humour as creativeness has been addressed, in both systematic review ${ }^{8}$ and elsewhere. Having a good sense of humour is thought to be a healthy and desirable trait, and refers to the readiness to respond positively to potentially funny stimuli, to the ability to use comedy as a coping strategy, ${ }^{9}$ and to the tendency to laugh and make others laugh. ${ }^{10}$ Incongruity theories describe humour as a creative process in which two normally disparate concepts or situations need to be brought together in an unexpected or incongruous manner. That is, humour arises when an idea or concept is suddenly viewed from an unusual perspective. Koestler coined the term 'bisociation' to describe the juxtaposition of two typically incongruous frames of references, which are placed together to create humour. ${ }^{11}$ These theories suggest that humour involves 'sudden, surprising shifts in the processing of information.' ${ }^{12}$ The creative elements needed to produce humour are strikingly similar to those characterising the cognitive style of people with psychosis (both schizophrenia and bipolar disorder).

Cognition in full-blown schizophrenia is associated with confusion, disorganisation, fragmentation, and thought and speech containing illogically connected ideas - the form of thought disorder known as 'overinclusive thinking. ${ }^{13}$ It is unsurprising, therefore, that people in an acute schizophrenic state have difficulty generating and understanding humour. ${ }^{14}$ However, in a more muted form overinclusive thinking is conceptually similar to divergent thinking, a term often applied in general psychology to explain creative thinking. This helps to resolve the apparent paradox that, although schizophrenic psychosis itself may be detrimental to humour, in its lesser form - as schizotypal personality - it might reflect a heightened disposition to promote humour, through a greater ability to associate odd or unusual things: in popular parlance to 'think outside the box'.

The picture for bipolar disorder is somewhat more straightforward, at least with respect to its mania side. Manic thinking is manifestly overinclusive (divergent); or what in this context has been described as combinatorial - defined as the ability to 'combine ideas or categories of thought in order to form new and original connections. ${ }^{15}$ Such thinking is much more pronounced among individuals with bipolar disorder than in those with schizophrenia, or in controls. ${ }^{16}$ It is easy to see how this can account for the relationship between the manic side of bipolar disorder and comic performance, facilitated through a synergism of very high mood and rapidly changing ideation. A notable example here is the English comedian Spike Milligan, who experienced manic-depressive episodes throughout his life. $^{17}$ Milligan certainly used the freely associating thought processes of his manic states to generate the zany humour and the wildly ridiculous ideas that were the hallmark of his comedy. Depression, however, with its slower thinking and anhedonia seems incompatible with creativity. Nevertheless, in the talented it might 
motivate the person to find ways of alleviating the low mood - in other words, to act as a form of self-medication. Pursuing this theme, Hugelshofer et al suggested that humour actually provides protection against the development of hopelessness and consequent depression. ${ }^{18}$ Being creative - writing, composing, painting and being humorous - might therefore be an outlet, an escape from the pain of depression. The poet and writer Antonin Artaud, who himself experienced serious mental illness, wrote, 'No one has ever written, painted or sculpted, modelled, built or invented except literally to get out of hell. ${ }^{19}$ Against this background we set out to test the hypothesis that comedians would resemble other creative individuals in showing a higher level of psychotic characteristics, both schizophrenic and manic depressive. For reasons intimated earlier, these effects should best be revealed by examining differences on measures of personality traits relating to psychosis, rather than in the clinical characteristics of psychosis itself. To this end we examined a large group of comedians on the short version of the Oxford-Liverpool Inventory of Feelings and Experiences (O-LIFE), a four-scale self-report inventory covering both schizophrenic and bipolar features. ${ }^{20}$ This instrument has been widely used in previous research on creativity. ${ }^{21,22}$ As controls we used another group of performing artists, namely actors. To establish their personality status relative to the general population, both of these groups were compared with the normative data for the O-LIFE questionnaire.

\section{Method}

Participants were recruited online and asked to fill in an online questionnaire. Recruitment of comedians was carried out by emails to online comedian agencies, comedy clubs, comedian associations and comedian societies mainly in the UK, USA and Australia; these were found by a Google search or on recommendation from other comedian or comedy societies. Comedy societies affiliated with UK universities were also contacted, as were comedy societies found on the social network Facebook. In a similar way the questionnaire was also sent to online acting societies, clubs and associations in the UK, USA and Australia, and to actors affiliated with UK universities, by email or Facebook. As part of the questionnaire, participants were asked to supply other basic information: age and gender, type of comedian (or actor), amateur or professional status, and length of time they had been performing. Participants were given the opportunity to receive their individual O-LIFE scores once the questionnaire was completed, as an encouragement to take part. Otherwise, it was emphasised that the survey was strictly anonymous.

\section{Questionnaire}

The questionnaire administered included all the items from the short version of the O-LIFE. ${ }^{20}$ This consists of four scales measuring different aspects of schizotypy/psychoticism, as follows:

(a) Unusual Experiences (UnEx), measuring magical thinking, belief in telepathy and other paranormal events, and a tendency to experience perceptual aberrations;

(b) Cognitive Disorganisation (CogDis), measuring distractibility and difficulty in focusing thoughts;

(c) Introvertive Anhedonia (IntAn), measuring a reduced ability to feel social and physical pleasure, including an avoidance of intimacy;

(d) Impulsive Non-conformity (ImpNon), measuring a tendency towards impulsive, antisocial behaviour, often suggesting a lack of mood-related self-control.

General population O-LIFE scores used for comparison formed part of the normative data for the short O-LIFE, obtained from a variety of studies of the questionnaire, and supplied by Oliver Mason (personal communication, 2012).

\section{Results}

The target sample of comedians consisted of 523 individuals (404 men and 119 women) with a mean age of 31.31 years (s.d. =9.77). The control sample comprised 364 actors (153 men and 211 women) whose mean age was 30.45 years $($ s.d. $=13.32)$. These samples were designated the 'performance groups'. The norms group consisted of 831 people ( 246 men and 585 women), with a mean age of 30.70 years (s.d. $=6.10)$. Although there was an imbalance for gender across the three groups, there was no significant overall difference in age: one-way analysis of variance (ANOVA) $F=1.13$, NS. Comparing the two performance groups on other variables, $57 \%$ of the comedians and $73 \%$ of the actors were amateurs, and time in their respective professions fell into similar bands: comedians $1-3$ years $54 \%, 4-7$ years $46 \%$; actors $1-3$ years $70 \%$, 4-7 years $30 \%$. As for type of comedian (or actor), both groups were offered several (not mutually exclusive) options to choose from, but in each case one form predominated. Thus, among comedians $85 \%$ described themselves as 'stand-up', with only a small degree of overlap with the other categories offered, of 'writer', 'sketch' and 'musical'. Similarly for actors: 94\% named 'theatre' as their preferred genre, with relatively few choosing 'musical', 'pantomime' or 'circus'. The exception there was that most also indicated that they worked in film.

Table 1 Scores on the Oxford-Liverpool Inventory of Feelings and Experiences for the two performance groups and normative data

\begin{tabular}{|lcccc} 
& \multicolumn{5}{c}{ Score, mean (s.d.) } \\
\cline { 2 - 5 } & IntAn & UnEx & CogDis & ImpNon \\
Comedians & & & & \\
$\quad$ Men $(n=404)$ & $3.09(2.10)$ & $4.55(2.89)$ & $5.75(2.77)$ & $4.57(2.22)$ \\
Women $(n=119)$ & $2.75(2.26)$ & $5.18(2.65)$ & $6.22(2.68)$ & $5.08(2.11)$ \\
Total $(n=523)$ & $3.01(2.14)$ & $4.70(2.85)$ & $5.86(2.75)$ & $4.68(2.21)$ \\
\hline Actors & & & \\
$\quad$ Men $(n=153)$ & $2.62(1.95)$ & $4.35(2.98)$ & $5.37(2.94)$ & $4.04(2.46)$ \\
Women $(n=211)$ & $2.10(1.85)$ & $4.97(2.82)$ & $5.67(2.64)$ & $4.60(2.30)$ \\
Total $(n=364)$ & $2.32(1.91)$ & $4.71(2.90)$ & $5.54(2.77)$ & $4.37(2.38)$ \\
\hline Norms & & & & \\
Men $(n=246)$ & $2.39(2.10)$ & $3.20(2.77)$ & $4.32(2.86)$ & $3.37(2.11)$ \\
Women $(n=585)$ & $2.08(1.89)$ & $3.84(3.12)$ & $5.14(2.89)$ & $3.61(2.08)$ \\
Total $(n=831)$ & $2.17(1.96)$ & $3.65(3.03)$ & $4.89(2.91)$ & $3.54(2.09)$ \\
CogDis, Cognitive Disorganisation; ImpNon, Impulsive Non-conformity; IntAn, Introvertive Anhedonia; UnEx, Unusual Experiences. & & \\
\hline
\end{tabular}




\begin{tabular}{|c|c|c|c|c|c|}
\hline & Sum of squares & d.f. & Mean square & $F$ & Significance \\
\hline \multicolumn{6}{|l|}{ Group } \\
\hline IntAn & 115.05 & 2 & 57.53 & 14.40 & $P<0.0001$ \\
\hline UnEx & 559.05 & 2 & 279.53 & 32.43 & $P<0.0001$ \\
\hline CogDis & 414.17 & 2 & 207.09 & 26.09 & $P<0.0001$ \\
\hline ImpNon & 465.38 & 2 & 232.69 & 48.82 & $P<0.0001$ \\
\hline \multicolumn{6}{|l|}{ Gender } \\
\hline IntAn & 49.63 & 1 & 49.63 & 12.42 & $P<0.0001$ \\
\hline UnEx & 127.79 & 1 & 127.79 & 14.83 & $P<0.0001$ \\
\hline CogDis & 88.61 & 1 & 88.61 & 11.17 & $P=0.001$ \\
\hline ImpNon & 61.45 & 1 & 61.45 & 12.89 & $P<0.0001$ \\
\hline \multicolumn{6}{|c|}{ Group $\times$ gender } \\
\hline IntAn & 2.67 & 2 & 1.34 & 0.33 & $P=0.72$ \\
\hline UnEx & 0.03 & 2 & 0.01 & 0.00 & $P=0.10$ \\
\hline CogDis & 17.83 & 2 & 8.92 & 1.12 & $P=0.33$ \\
\hline ImpNon & 7.73 & 2 & 3.87 & 0.81 & $P=0.44$ \\
\hline
\end{tabular}

\section{O-LIFE scores}

Table 1 shows the mean scores for the three groups on the four O-LIFE scales. These data were analysed by multivariate analysis of variance (Table 2). There was a highly significant difference across the norms group and the two performance groups. Consistent with the norms for the O-LIFE there was also a significant gender difference, but no gender $\times$ group interaction. Gender was therefore not considered further at this stage of the analysis. Considering pairwise comparisons between groups (Tukey test), both comedians and actors had significantly higher O-LIFE scores than the test norms on all scales $(P<0.001)$, with the exception that actors did not differ from the norms on IntAn $(P<0.35)$. Actors also scored significantly lower than comedians on that scale $(P<0.001)$, as well as on $\operatorname{CogDis}(P<0.026)$ and ImpNon $(P<0.02)$. However, the performance groups scored equally highly on UnEx.

The pattern of O-LIFE scores observed in the two performance groups prompted us to look more closely at their respective profiles across the four questionnaire scales. To conduct this comparison the O-LIFE scores for participants in those two

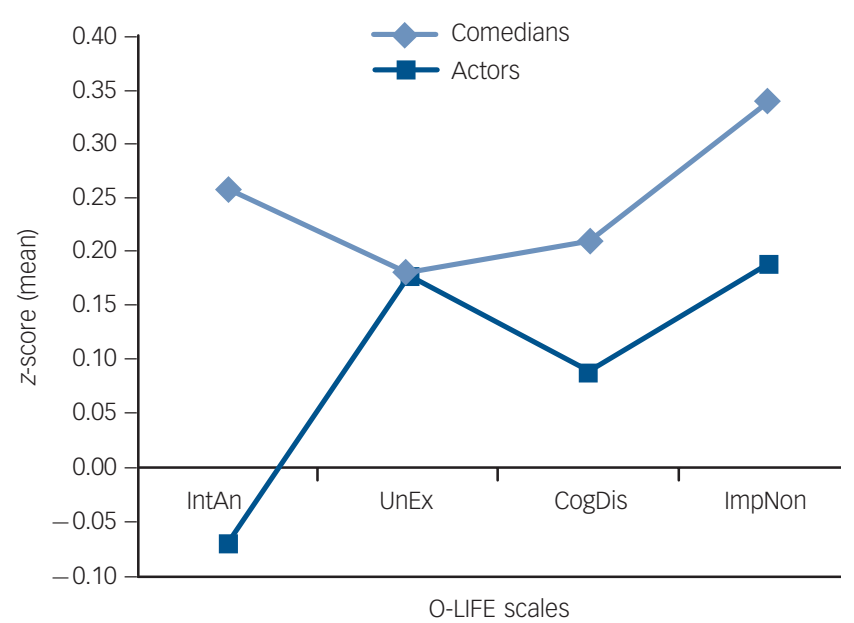

Fig. 1 Comedians' and actors' profiles on the four scales of the Oxford-Liverpool Inventory of Feelings and Experiences (O-LIFE). CogDis, Cognitive Disorganisation; IntAn, Introvertive Anhedonia; ImpNon, Impulsive Non-conformity; UnEx, Unusual Experiences. groups were converted into $z$-scores. The two groups showed markedly different profiles (Fig. 1), particularly defined by the high IntAn and ImpNon scores observed in comedians compared with actors. Further inspection of these profile data revealed some gender differences (Fig. 2). Two things stand out: one is that the overall profile for the comedians group is even more exaggerated in women, and the second is the striking gender difference among the actors group, women scoring notably low on IntAn yet high on ImpNon, mirroring and accounting for the overall trend in that group. Male participants, in comparison, had a flat, unremarkable profile across all four scales.

\section{Discussion}

The results of this study substantially confirmed our expectation that comedians would behave like other creative groups in showing a high level of psychotic personality traits. They did so across all the domains sampled by the questionnaire we used, from schizoid and schizophrenic-like characteristics through to manic-depressive features. The most striking result, however, was the comedians'

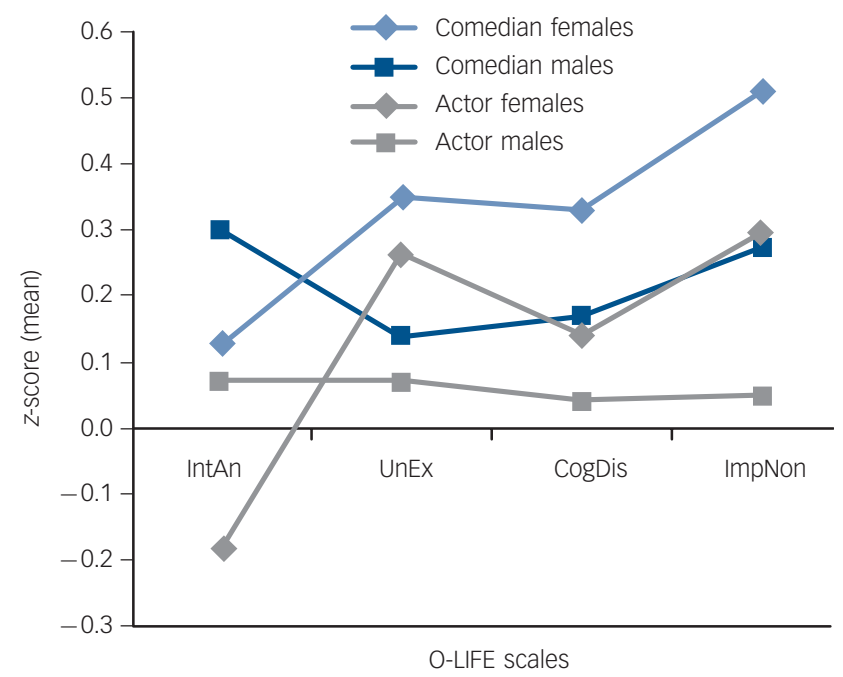

Fig. 2 Gender differences in comedians' and actors' profiles on the four scales of the Oxford-Liverpool Inventory of Feelings and Experiences (O-LIFE). CogDis, Cognitive Disorganisation; IntAn, Introvertive Anhedonia; ImpNon, Impulsive Non-conformity; UnEx, Unusual Experiences. 
unusual personality profile observed across the four O-LIFE scales, scoring highly on both IntAn and ImpNon. This is of interest because the scales in question tap seemingly opposite personality characteristics: unsociable, depressive traits in the case of IntAn and more extraverted, manic-like traits in the case of ImpNon. We believe that the profile represents the personality equivalent of bipolar disorder, corresponding to what in an older personality literature would have been termed 'cyclothymic temperament'. This is illustrated in the self-description given by one of our (male) comedian respondents:

'I can say about myself that I don't think I'm one specific way. Sometimes I'm extremely introverted or uncomfortable socially and other times I'm the life of the party type of person. I believe though that if I'm in a situation where I might feel judgement for my inability to be/act "normal", either founded by paranoia or legitimacy, I'm more likely to act out/overcompensate in the "not afraid what anyone thinks" department.

Existing alongside high ratings on the two cognitive scales of the O-LIFE - UnEx and CogDis, both of which measure out-of-theordinary ways of thinking - it is possible to see how these various features combine synergistically to facilitate comedic performance. Or, as another of our respondents put it:

'Comedians train their brains to think in wide associative patterns. This relates to joke writing, where the word "bicycle" brings up a picture of a bicycle in the mind of a non-comedian, but for the comedian it's like running a search on the internet - everything related pops up, from images of fat people riding bicycles naked and getting chafed to the fact that Lance Armstrong only has one testicle ... I agree that some disorders have the helpful attribute of removing filters. Bipolar is usually hyperverbal, and means that focus keeps shifting, the way it must to absorb everything at once.'

The O-LIFE factors are regarded conceptually as trait dimensions, intended as descriptors of predispositions to disorder - in the case of manic-depressive traits the tendency to experience consecutive changes in mood. Yet it is also helpful to take a slightly different, state, perspective on the matter, to see whether this gives any further insight into the psychology of the comedian; in other words to ask how (especially) the affective traits, shown to be important here, play out in the actual comedic situation. As it happens, when we were writing this paper a news item addressing the issue appeared across the British media about and by the celebrated English comedian Stephen Fry. ${ }^{23}$ Fry, who has a diagnosis of bipolar disorder (by his own reckoning 'bipolar lite') and is currently president of the mental health charity Mind, recently confessed to a suicide bid he had made and, in the course of talking about it, elaborated further on a typical mental state he had while performing. In the following, Fry is referring to this in the context of his role as the jokey compère of BBC television's highbrow quiz show, QI:

'There are times when I'm doing QI and I'm going "ha ha, yeah, yeah", and inside I'm going "I want to fucking die. I . . . want . . to . . . fucking . . die".'

Fry's comment (expressed in characteristically robust fashion) serves to illustrate how two conflicting emotional traits typically found in comedians might be evoked simultaneously to shape their behaviour, one being used to cope with the other. There is something reminiscent here of the psychoanalytic notion of the 'manic defence.' 24

Using actors as controls for our comedian sample proved informative in several ways. It showed that although both groups had performing in front of an audience in common, they had distinctly different personality profiles. Most notably, the actors were low in the introversion traits measured by the IntAn scale, while sharing with comedians the more extraverted traits measured by the ImpNon scale. These personality profiles could explain how each relates to their respective audiences, and what motivates them to do so. The high IntAn rating in the comedians group suggests that, at one level, such individuals are indifferent to the audience; only their simultaneous disposition to the extraversion that forms part of ImpNon perhaps allows engagement to occur in public performance. Actors, on the other hand, being more congruent in their introvert/extravert tendencies, might be motivated towards a more straightforward, 'genuine' engagement with an audience, as acting seems to demand.

The results presented here convincingly demonstrate that, as creative people, comedians rate highly on the same personality traits as those regularly observed in other creative individuals. The traits in question are rightly labelled 'psychotic' because they represent healthy equivalents of cognitive and temperamental variations which, in pathological form, predispose to and mediate the symptoms of psychotic illness: features such as moodiness, social introversion and the tendency to lateral thinking. Humour and the conditions for it are particularly good examples of this 'madness/creativity' connection and deserve more attention than they have received hitherto. Of particular interest would be to supplement psychometric studies of the kind reported here with more detailed, biographical investigations such as those undertaken in other parts of the creativity literature.

\section{Limitations}

Although the use of an online methodology allowed us to collect a substantial sample of participants, a limitation of the study was that it did not enable us to assess the response rate of those surveyed, or to judge the representativeness of the samples. In addition, refinement of the method would have made it possible to examine differences between subtypes of comedians (and actors), most of whom reported themselves as falling into one type. A particular consequence of this was that we were unable to assess the importance of the fact that comedians (mostly) write their own material and actors (mostly) do not.

\section{Victoria Ando, BA, Gordon Claridge, DSC, Department of Experimental Psychology, University of Oxford, Oxford; Ken Clark, BA, Department of Psychology, Berkshire} Healthcare NHS Foundation Trust, Reading, UK

Correspondence: Victoria Ando, St Hugh's College, University of Oxford, oxford OX2 6LE, UK. Email: victoria.ando@new.oxon.org

First received 21 Jul 2013, final revision 10 Sep 2013, accepted 13 Nov 2013

\section{Acknowledgements}

We thank Oliver Mason for supplying updated normative data for the Oxford-Liverpool Inventory of Feelings and Experiences and Stephen Thompson and Christian Ancliffe for their invaluable help with the recruitment of many of the comedians who participated in the study.

\section{References}

1 Auster P The Notebooks of Joseph Joubert: A Selection. New York Review of Books Classics, 2006 [Joseph Joubert 1754-1824, no original work published during his life].

2 Silvia P, Kaufman J. Creativity and mental illness. In The Cambridge Handbook of Creativity (eds JC Kaufman, RJ Sternberg): 381-94. Cambridge University Press, 2010.

3 Brod JH. Creativity and schizotypy. In Schizotypy: Implications for IIIness and Health (ed. G Claridge): 274-99. Oxford University Press, 1997.

4 Claridge G, Barrantes-Vidal N. Creativity: a healthy side of madness. In Chimes of Time: Essays by Wounded Professionals (ed. B Kirkaldy): 115-30. Sidestone, 2013.

5 McBride AJ. Comedians: fun and dysfunctionality. Br J Psychiatry 2004; 185 177.

6 Rawlings D. Relating humor preference to schizotypy and autism scores in a student sample. Humor 2008; 21: 197-219.

7 Greengross G, Miller GF. The Big Five personality traits of professional comedians compared to amateur comedians, comedy writers and college students. Pers Indiv Dif 2009; 47: 79-83. 
8 O'Quin K, Derks P. Humor. In Encyclopedia of Creativity (Vol. 1) (eds M Runco, SR Pritzker): 845-52. Academic Press, 1999.

9 Chapman AJ, Foot HC (eds). Humour and Laughter: Theory, Research and Applications. Pitman, 1976

10 Forabosco G. The ill side of humour: pathological conditions and sense of humour. In The Sense of Humour: Explorations of a Personality Characteristic (ed. W Ruch): 271-92. Walter de Gruyter, 1998.

11 Koestler A. The Act of Creation. Hutchinson, 1964

12 Lefcourt HM, Martin RA. Humor and Life Stress: Antidote to Adversity. springer, 1986.

13 Cameron N. The functional psychoses. In Personality and the Behavior Disorders, vol 2 (ed. JMcV Hunt): 861-921. Ronald Press, 1944.

14 Marjoram D, Tansley H, Miller P, Macintyre D, Owens DG, Johnstone EC, et al. $A$ theory of mind investigation into the appreciation of visual jokes in schizophrenia. BMC Psychiatry 2005; 5: 12.

15 Jamison KR. Touched with Fire: Manic-Depressive Illness and the Artistic Temperament. Free Press, 1993.
16 Solovay MR, Shenton ME, Holzman PS. Comparative studies of thought disorder: I. Mania and schizophrenia. Arch Gen Psychiatry 1987; 44: 13-20.

17 Milligan S, Clare A. Depression and How To Survive It. Ebury Press, 1993.

18 Hugelshofer DS, Kwon P, Reff RC, Olson ML. Humour's role in the relation between attributional style and dysphoria. Eur J Pers 2006; 20 : 325-36.

19 Artaud A. Van Gogh, the man suicided by society. In Antonin Arnaud: Selected Writings (ed. S Sontag). University of California Press, 1992.

20 Mason O, Linney $Y$, Claridge G. Short scales for measuring schizotypy. Schizophr Res 2005; 78: 293-6.

21 Nettle D. Schizotypy and mental health amongst poets, visual artists and mathematicians. J Res Personality 2006; 40: 276-90.

22 Claridge $\mathrm{G}$ (ed). Personality, psychopathology, and original minds. Pers Indiv Dif 2009; 46 (special issue): 749-838.

23 Sherwin A. Stephen Fry reveals he attempted suicide in 2012. Independent 2013; 6 June.

23 Klein M. Mourning and its relation to manic-depressive states. Int J Psychoanalysis 1940; 21: 125-53.

\section{reflection}

\section{Social Origins of Depression, by George W. Brown \& Tirril Harris}

\section{Julian Leff}

When I started my training in psychiatry at the Maudsley Hospital I was taught that there were two types of depression, reactive and endogenous, the latter being uninfluenced by environmental factors. This distinction has faded into history, partly due to the research of George Brown and Tyrril Harris on life events and depression. Their work was foreshadowed by Holmes and Rahe who studied the relationship between life events and the development of schizophrenia in conscripts to the US Navy. Their measure of the impact of events was insufficiently objective and was superseded by the rigorous work of Brown and Harris, who inquired in detail into the individual circumstances of each person who reported an event. They also classified events as independent of the person's behaviour or not in an attempt to clarify the issue of causality. They focused on women and found that those who had a supportive partner were much less likely to develop depression than those who lacked an intimate relationship. Their research on depression continued over several decades with increasing sophistication and led to the conclusion that events preceding episodes of depression entailed a major loss, whereas episodes of anxiety were precipitated by events that threatened losses of various kinds. In recent years they addressed the issue of causality by initiating a randomised controlled trial evaluating the efficacy of providing a female befriender for women who lacked a supportive partner. This intervention proved to protect vulnerable women from the impact of life events.

Prior to his work on depression, Brown collaborated with Michael Rutter on developing a measure of the emotional relationship between carers and their relatives with schizophrenia, which they named expressed emotion (EE). This proved to be a remarkably potent predictor of relapse in schizophrenia and also in depression, as shown by Christine Vaughn and myself. After years of focusing on schizophrenia I felt I should return to studying depression, and mounted an intervention trial for depressed patients living with a critical partner. This was a parallel to Brown and Harris's trial of befriending, but instead of providing a supportive partner, we employed two couple therapists to attempt to improve the relationship between the patient and her/his partner. We found that couple therapy was more effective than antidepressants in treating depression and preventing relapse, and much more acceptable to the clients. Furthermore, the improvements in depression were attributable to reduction in the patient's exposure to hostility shown by their partner.

If, as is often stated, psychiatry is the cinderella of medicine, then social psychiatry, with its focus on human relationships, is, in the view of many biological psychiatrists, the Cinderella of psychiatry. It was George Brown who waved the fairy godmother's wand and transformed this ragged and despised subspecialty into a substantial discipline, capable of developing innovative and efficacious treatments, which equal or even surpass the achievements of biological psychiatry, and in the case of family work for schizophrenia have been incorporated in NICE guidelines. 